damit ungesunden Fettsäuren reichen Cholesterinestern um 8,8\%. Wichtig ist: Lactobacillus reuteri NCIMB 30242 muss enthalten sein! Die Kapseln werden in den USA übrigens sinnigerweise von einer Firma namens Micropharma als "Cardioviva“ angeboten. Lang lebe das Herz.

- Jones ML, Abstract 11348

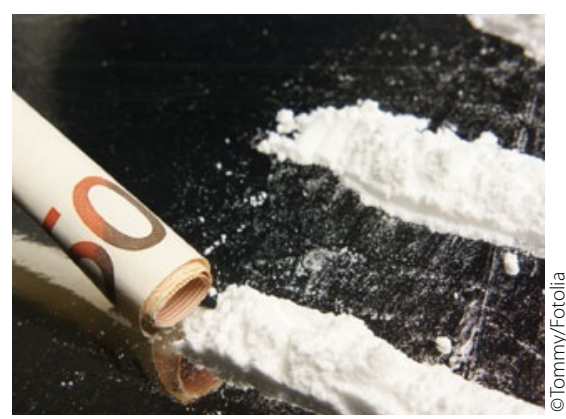

Herzinfarktdroge.

\section{Wie Kokainschnupfer ihre Gefäße ruinieren}

- Eine Prise Kokain schnupfen, für manche ist das der ultimative Partykick. Doch bei regelmäßigem Konsum droht dem Gefäßsystem ein dauerhafter Schaden, berichten Autoren aus Sydney. Ihnen war es gelungen, 20 chronische Kokainkonsumenten durchzuchecken und dabei auch in einen Magnetresonanztomografen zu bugsieren. Was sie fanden war: Eine um 30-35\% erhöhte Aortensteifigkeit, im Schnitt um 18\% verbreiterte linksventrikuläre Wände und einen um $8 \mathrm{mmHg}$ erhöhten Blutdruck im Vergleich zu Kontrollen, die ohne Kokain durchs Leben kamen.

Schade eigentlich, sagte Gemma Figtree, Universität Sidney, die Studienautorin: Wir sehen immer wieder fitte junge Menschen mit massiven Herzinfarkten, weil sie Kokain einnehmen. Kokain ist die perfekte Herzinfarktdroge, sagte sie.

DR. MED. DIRK EINECKE ॥

- Figtree G, Abstract 18163

Quelle: Alle Abstacts der Scientific Sessions 2012 der American Heart Association finden Sie hier : http://www.abstractsonline.com/plan/start.aspx ?mkey=\%7B14145D5B\%2DF96B\%2D4354\%2D8 237\%2D8F0937744BA4\%7D

Relax-AHF

\title{
Endlich eine positive Studie bei akuter Herzinsuffizienz
}

\section{Mit dem Schwangerschaftshormon Relaxin deutet sich zum ersten Mal seit langer Zeit ein Fortschritt in der Behandlung der akuten Herzinsuffi- zienz an: In der RELAX-AHF-Studie verbesserte die Therapie die Atemnot der Patienten sowie die Überlebenschancen nach sechs Monaten.}

- In der internationale Doppelblindstudie waren 1161 Patienten aufgenommen worden, die mit akuter Herzinsuffizienz ins Krankenhaus kamen. Zu den Einschlusskriterien gehörten deutliche Atemnot, Kongestion, mäßige Niereninsuffizienz, und ein stabiler Blutdruck über $125 \mathrm{mmHg}$. Die Behandlung erfolgte mit rekombinantem humanen Relaxin-2 (Serelaxin) binnen 16 Stunden nach der Aufnahme mittels Infusion über 48 Stunden in einer Dosierung von $30 \mu \mathrm{g} / \mathrm{kg} / \mathrm{d}$.

\section{Akute Atemnot gelindert}

Es gab zwei Co-primäre Endpunkte, welche die Atemnot quantifizierten. Einer dieser Endpunkte, die mit Hilfe der visuellen Analogskala beurteilte Dyspnoe innerhalb der ersten fünf Tage, verbesserte sich signifikant: Die Fläche unter der Kurve wurde durch Serelaxin um $19,4 \%$ vergrößert $(p=0,0075)$. Dies bedeutet, dass die Patienten eine Erleichterung der Atemnot verspürten, so Studienautor Prof. John R. Teerlink, San Francisco VA Medical Center.

\section{Kein Einfluss auf erneute}

\section{Krankenhauseinweisungen}

Nicht signifikant gebessert wurde die kardiovaskuläre Mortalität oder die Rehospitalisierungsrate innerhalb von 60 Tagen - beides sekundäre Endpunkte. Patienten der Verumgruppe verzeichneten andererseits signifikant seltener eine Verschlechterung der Herzschwä- che. Sie benötigten weniger intravenöse Diuretika, und auch die Zeichen der Kongestion bildeten sich deutlicher zurück als in der Kontrollgruppe. Patienten der Verumgruppe wurden im Schnitt einen Tag früher aus dem Krankenhaus entlassen.

\section{Gesamtmortalität nach einem} halben Jahr um $37 \%$ reduziert

Positiv überraschte vor allem der Befund, dass nach 180 Tagen 65 Patienten $(11,3 \%)$ in der Placebogruppe verstorben waren, aber nur 42 Patienten in der Verumgruppe (7,3\%). Dies entspricht einer relativen Risikosenkung von $37 \%(p=0,02)$. Die NNT, um einen Todesfall zu verhindern, beträgt 29 , so Teerlink.

Relaxin ist ein Hormon mit antiischämischen, antiinflammatorischen und antifibrotischen Eigenschaften. Während einer Schwangerschaft sorgt es für die physiologischen hämodynamischen Veränderungen, die notwendig sind, um den Fötus mitzuversorgen. Dazu zählen eine 20\%ige Erhöhung der kardialen Auswurfleistung, eine 50-85\%ige Erhöhung der Nierendurchblutung, eine $40-65 \%$ ige Verbesserung der Kreatinin-Clearance, oder eine $30 \%$ ige Senkung der systemischen vaskulären Resistenz. In einer kleineren Phase-2-Studie war Serelaxin bei akuter Herzschwäche ebenfalls symptomatisch und prognostisch wirksam gewesen.

DR. MED. DIRK EINECKE -

\footnotetext{
- Quelle: Jahrestagung der American Heart Association, Los Angeles, 4.11.-7.11.2012. Teerlink JR, Cotter G, et al.: Serelaxin, recombinant human relaxin-2, for treatment of acute heart failure (RELAX-AHF): a randomised, placebo-controlled trial. The Lancet 2012, DOI: 10.1016/S0140-6736(12)61855-8
} 